\title{
Cardiac Biomarkers and the Diagnosis of Myocardial Infarction in Women
}

\author{
Anoop S. V. Shah ${ }^{1} \cdot$ Amy V. Ferry ${ }^{1} \cdot$ Nicholas L. Mills ${ }^{1}$
}

Published online: 8 April 2017

(C) The Author(s) 2017. This article is published with open access at Springerlink.com

\begin{abstract}
Purpose of review Women with suspected acute coronary syndrome are less likely to undergo investigation or receive treatment than men, and women consistently have poorer outcomes. This review summarises how the latest development in cardiac biomarkers could improve both diagnosis and outcomes in women.

Recent findings Novel high-sensitivity cardiac troponin assays have identified differences in the reference range and therefore diagnostic threshold for myocardial infarction in men and women. These differences are present across multiple populations with different ethnic backgrounds and for a range of assays. The use of a uniform threshold for cardiac troponin does not provide equivalent prediction in men and women, with lower thresholds needed for women to provide comparable risk stratification.

Summary Sex differences in cardiac troponin concentrations are not widely recognised in clinical practice and may be contributing to the under-diagnosis of myocardial infarction in women and discrepancies in patient care and outcomes.
\end{abstract}

Keywords Sex $\cdot$ Cardiac troponin $\cdot$ Biomarkers $\cdot$ Myocardial infarction

This article is part of the Topical Collection on Management of Acute Coronary Syndromes

Anoop S. V. Shah

anoop.shah@ed.ac.uk

1 BHF Centre for Cardiovascular Science, University of Edinburgh, Edinburgh EH16 4SB, UK

\section{Introduction}

In September 2000, the United Nations Millennium Declaration committed to promote gender equality as one of their primary goals to achieve health equity. Globally, coronary heart disease remains the major cause of death in women in both high-and low- or middle-income nations [1]. Despite this, sex differences remain in multiple aspects of cardiovascular care including diagnosis, access to investigation and treatment and outcomes $[2 \bullet \bullet]$. Furthermore, these differences are prevalent even in highly developed health-care systems such as the USA $[2 \bullet \bullet, 3]$. The most recent scientific statement from the American Heart Association (AHA) highlights two key areas that contribute to sex differences: biological or social factors, and the underrepresentation of women in clinical trials [4].

Cardiovascular medicine is fortunate to have a high-quality evidence base derived from multiple randomised control trials to guide our practice. However, a comprehensive summary by Nanette Wenger highlights how medical research has neglected the health needs of women especially in cardiovascular medicine $[2 \bullet \bullet, 4]$. Whilst improvements have been made to increase recruitment of women in clinical trials, to date, large randomised control trials include a majority of men. As such, the evidence on safety and efficacy of key therapeutic interventions in acute myocardial infarction are limited in women with data largely derived from their male counterparts.

More concerning is the evidence from epidemiological studies and randomised controlled trials of disparities in outcomes following myocardial infarction in men and women [3, 5]. The AHA scientific statement on myocardial infarction in women highlighted this issue [4]. In data derived from large multi-centred randomised control trials, women consistently have higher case fatality rates compared to men, and these differences exist despite adjusting for age and comorbid conditions (Fig. 1a). Indeed, it is in the younger age groups that 
women are more likely than men to have an adverse event following myocardial infarction (Fig. 1b). In a consecutive series of over a million patients with acute coronary syndrome, even women $<45$ years were at increased risk of death compared to men of a similar age (relative risk [RR] 1.3 [95\% confidence interval 1.2 to 1.4]) [3]. It is less clear what biological or social factors are responsible for these disparities. Many have been proposed, including sex differences in the pathobiology of acute myocardial infarction, in the time taken to seek medical advice following the onset of symptoms and in the efficacy of treatment for myocardial infarction. There may also be differences in the presenting symptomology between men and women, which may result in misdiagnosis, delay in providing evidence-based therapy and explain the worse outcomes seen in women.

An alternative explanation, and one that is gaining increasing attention, is that we are under-diagnosing myocardial infarction in women by not recognizing that there are important differences in the reference range and diagnostic threshold of cardiac biomarkers between men and women.

\section{Diagnosis of Myocardial Infarction}

The diagnosis of myocardial infarction is based on symptoms or signs of myocardial ischaemia and biomarker evidence of myocardial necrosis. The definition is largely unchanged from the initial MONICA World Health Organization (WHO) statement. What has, however, changed significantly over the last few decades is our ability to detect and quantify myocardial necrosis. The aim of the original WHO definition was to standardise diagnosis and permit monitoring of trends and determinants of myocardial infarction in epidemiological studies. This definition was not considered sufficiently specific in clinical practice for the assessment of individual patients and was open to interpretation [10]. The need for a more precise definition of myocardial infarction coupled with the availability of more sensitive and specific biomarkers of myocardial necrosis resulted in a series of consensus statements and revisions of the definition of myocardial infarction.

Serum biomarkers have been used to assist in the diagnosis of acute myocardial infarction for over half a century with aspartate transaminases in the 1950s and lactate dehydrogenase in the 1970s [11]. However, it was not until the late 1970s when an isoenzyme of creatine kinase (CK-MB) was discovered that a biomarker revolutionised the clinical diagnosis of myocardial infarction. In 2003, Apple et al. evaluated seven CK-MB mass assays in 696 healthy participants, demonstrating that the 99th centile upper reference limit was two to threefold higher in men [12]. As such, the National Academy of Clinical Biochemistry guidelines advocated the use of sex-specific 99th centile upper reference limits when using CK-MB with a class $1 \mathrm{C}$ recommendation [13].

\section{Cardiac Troponins}

Troponin is a 3-piece regulatory protein complex present in cardiac and skeletal muscle and is integral to muscle contraction. Both cardiac troponin $\mathrm{T}$ and troponin I are derived from genes specific to the myocardium $[14,15]$, whereas troponin $\mathrm{C}$ is present in both cardiac and skeletal muscles with no specific isoform for cardiac tissue [14].

Given the high specificity of cardiac troponin $\mathrm{T}$ and troponin I to the myocardium, quantification of these biomarkers has now become integral to the diagnosis of myocardial infarction. The latest consensus statement defines myocardial infarction as a rise and/or fall in cardiac troponin with at least one value above the 99th centile upper reference limit in the context of symptoms or clinical evidence of myocardial ischaemia [16]. This was a paradigm shift replacing CK-MB as the gold standard for the diagnosis of myocardial infarction.

Over the last decade, troponin assays have become increasingly sensitive, from the older generation conventional assays to the more sensitive contemporary troponin assays. The latest developments in assay technology have led to the increasing use of high-sensitivity troponin assays. These assays have been defined as high-sensitivity by the International Federation of Clinical Chemistry Task Force based on assay performance [17]. A high-sensitive assay should meet two basic criteria: first, the total coefficient of variation (a measure of [im]precision) should be $\leq 10 \%$ at the 99th centile of a healthy reference population and, second, at least $50 \%$ (and ideally $90 \%$ ) of the reference population should have a measurable troponin concentration above the limit of detection.

\section{Sex Differences in Cardiac Troponin Concentrations}

The older generation cardiac troponin assays had initially been thought to be good gender-independent markers for diagnosis in acute coronary syndrome. Key papers published in the last two decades challenged this view. In the 1990s, Hamm et al. measured cardiac troponin $\mathrm{T}$ in men and women with unstable angina in whom acute myocardial infarction was excluded. The study showed that troponin $\mathrm{T}$ concentrations were measurable $(>200 \mathrm{ng} / \mathrm{L})$ in $43 \%$ of men and only $27 \%$ of women [18]. This initial observation was supported by Wiviott et al. who demonstrated that in patients with unstable angina or non-ST segment elevation myocardial infarction pooled from four randomised control trials, men were more likely to have elevated cardiac biomarkers including cardiac troponin I, cardiac troponin $\mathrm{T}$ and CK-MB compared to women using older generation assays with a single diagnostic threshold [19].

More recently, developments in assay technology have greatly enhanced sensitivity and, for the first time, have been able to quantify circulating troponin in the majority of individuals in a normal healthy reference population [20]. Measuring troponin using high-sensitivity assays has revealed important differences 
Fig. 1 Risk of mortality in women compared to men in randomised control trials before and after adjustment for age (a) and stratified by age in a large cohort study (b). TIMI II Thrombolysis in myocardial infarction II [6], GUSTO Global Utilization of t-PA and

Streptokinase for Occluded Coronary Arteries (GUSTO-I) trial [7], TAMI Thrombolysis and Angioplasty in Myocardial Infarction [8], ISIS-3 Third International Study of Infarct Survival [9] a

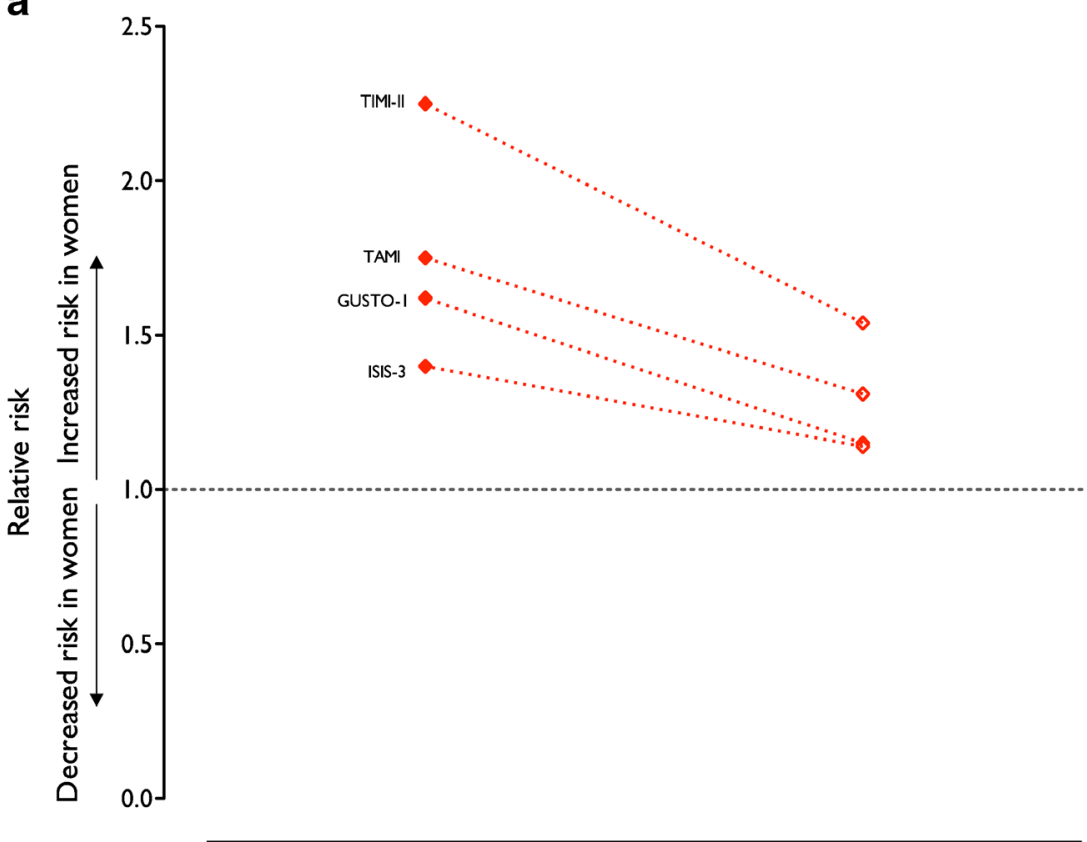

Crude RR

Age-adjusted RR

b

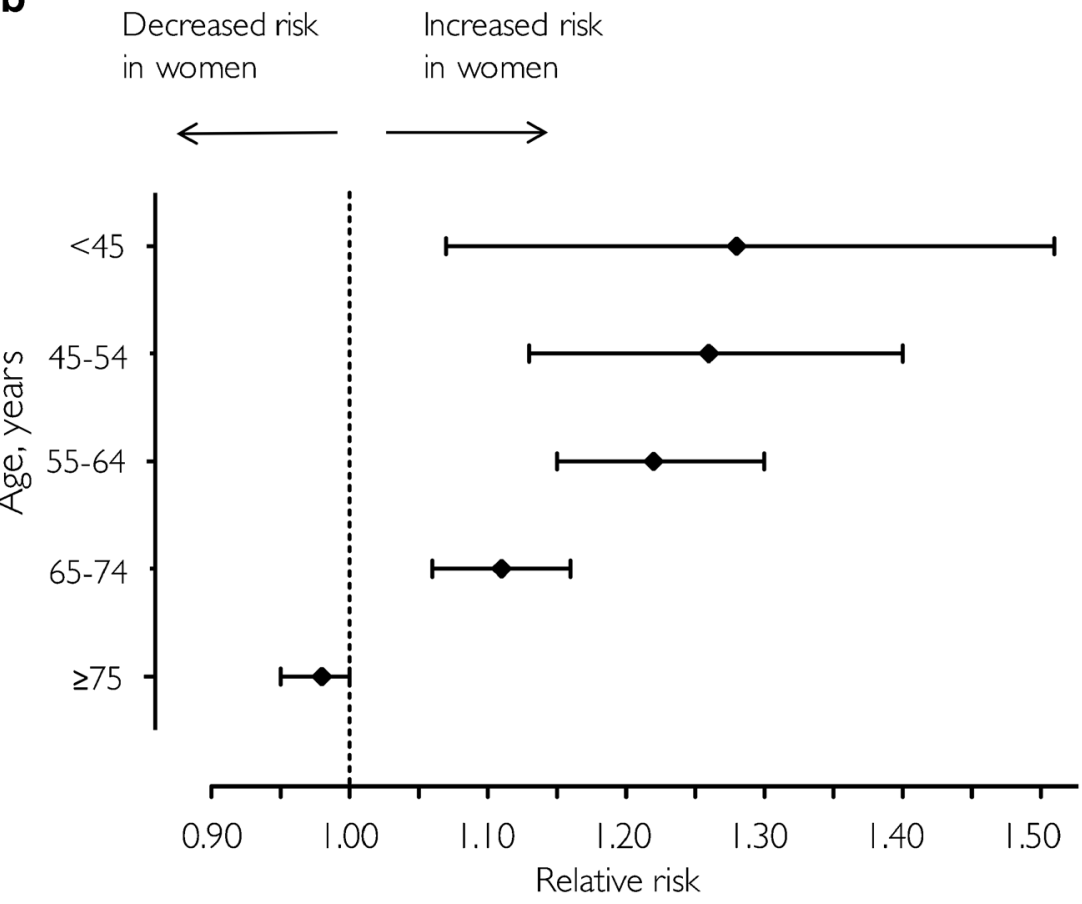

between men and women, with the 99th centile reference limits up to two-fold higher in men [20]. This observation has been consistent across all troponin assays that have been evaluated and has now been reported in multiple populations from different ethnic backgrounds (Table 1). Indeed, the reference range has been studied for 19 different assays in the same population, demonstrating higher 99th centile upper reference limits for the two clinically available high-sensitivity assays: cardiac troponin $\mathrm{T}$
(20 ng/L men, $13 \mathrm{ng} / \mathrm{L}$ women) and cardiac troponin I (36 ng/ L men, $15 \mathrm{ng} / \mathrm{L}$ women) [20].

The mechanisms through which cardiac troponin is released into the circulation in the apparent 'healthy' state is unclear, but may reflect cardiomyocyte apoptosis and cell turnover, hypertrophy or sub-clinical myocardial fibrosis $[29,30]$. Furthermore, it is unclear why there is a difference in the distribution of cardiac troponin concentrations in men 


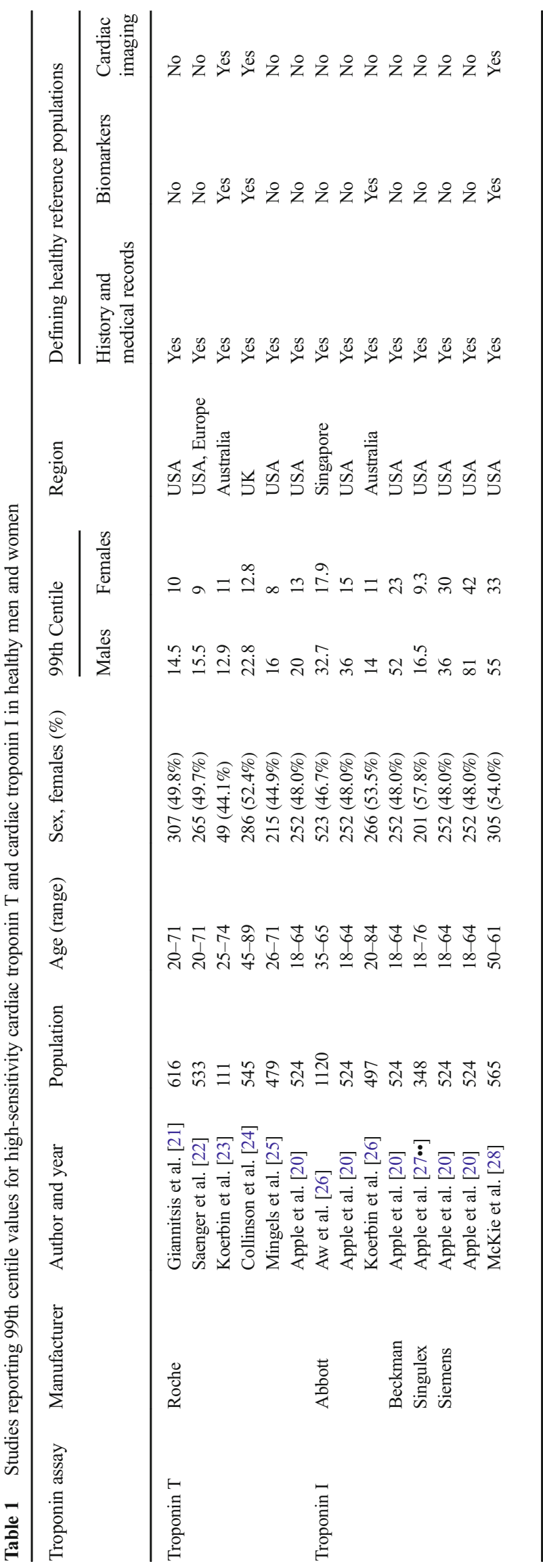

and women [31], but multiple hypotheses have been proposed (Fig. 2) [29, 32, 33]. In contrast, sex differences in CK-MB concentrations were attributed to differences in skeletal mass, which contributes $1-2 \%$ of circulating CK-MB [31] and is likely to explain higher levels in men. This is not the case for cardiac troponin, which has excellent tissue specificity, and therefore differences in the reference limits are likely to reflect differences in cardiovascular physiology or the prevalence of sub-clinical pathology in men and women.

Likewise, in acute coronary syndrome, women have lower cardiac troponin concentrations than men, perhaps suggesting differences in the mechanism of myocardial infarction between sexes. For example, in women with acute coronary syndrome enrolled in the TACTICS-TIMI 18 trial, the odds of having an elevated plasma cardiac troponin T or I concentrations were 0.53 [ $95 \%$ CI 0.43 to 0.68 ]) and 0.58 [95\% CI 0.46 to 0.73 ], respectively, compared to men [19]. Changes in reproductive hormones during the menstrual cycle, pregnancy and menopause influence both vasomotor function and endogenous fibrinolysis [34]. Perhaps, as a consequence, women with acute coronary syndrome are less likely to have evidence of plaque rupture (6.6 versus $16.3 \% ; P=0.002$ ) on intravascular coronary imaging studies [35]. Indeed, women are more likely than men to have non-obstructive atherosclerotic disease with either demonstrable vasospasm, spontaneous coronary artery dissection or plaque erosion with microembolization [36]; all of which are likely to result in less myocardial injury and lower cardiac biomarker concentrations.

\section{Should We Apply Sex-Specific Thresholds for the Diagnosis of Myocardial Infarction?}

Both inconvenience and a potential source of confusion for clinicians have been used as arguments against the use of sexspecific thresholds [37]. However, sex-specific reference ranges are not unfamiliar in clinical medicine and clinicians have coped with such scenarios for decades. Common laboratory parameters, including haemoglobin and glomerular filtration rate, account for sex when determining reference limits. Traditionally, clinical decision limits adjusted for sex have been solely based on reference range studies [12], but given the critical role of cardiac troponins in the diagnosis of myocardial infarction, additional evidence is required from outcome studies and from studies that evaluate diagnostic accuracy of sex-specific thresholds.

\section{Sex Differences in Troponin and Cardiovascular Outcomes}

Recently, a number of studies have evaluated differences in the prognostic utility of high-sensitivity cardiac troponin between men and women in stable populations. The Activity and Function in the Elderly study showed that in 1506 participants 
Fig. 2 Proposed biological explanations for differences in the distribution of cardiac troponin in men and women

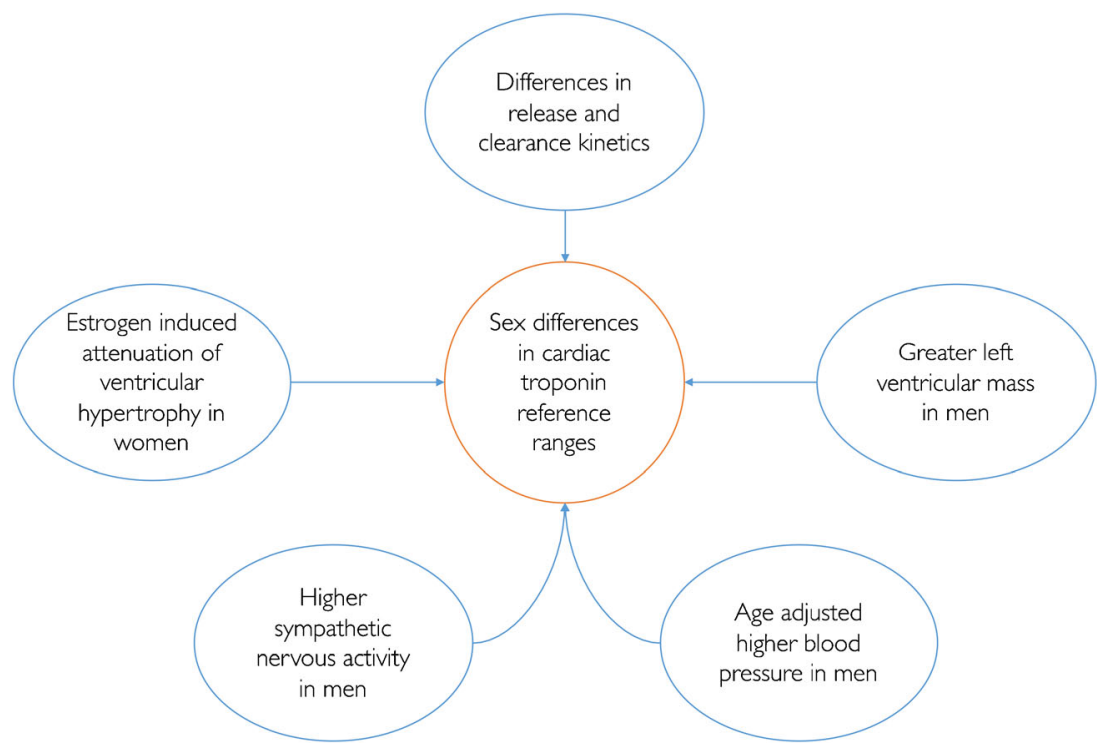

over the age of 65 years, increasing cardiac troponin $\mathrm{T}$ and troponin I concentrations were strongly associated with allcause mortality. These association varied by sex with women having numerically higher age-adjusted associations with mortality per unit increment in cardiac troponin (logtransformed) compared to men for both troponin T (RR 3.67 [95\% confidence interval (CI) 2.31 to 5.81] versus 2.15 [95\% CI 1.61 to 2.87]) and troponin I (3.33 [95\% CI 2.13 to 5.18] versus 1.92 [95\% CI 1.55 to 2.38]) [38]. Similar associations were echoed in the larger HUNT study with 9712 participants including 5281 (54\%) women. Increasing high-sensitivity cardiac troponin I concentrations were associated with all-cause mortality (RR 1.17 [95\% CI 1.12 to 1.22 ] per 1 SD increment in log troponin) and cardiovascular death (1.23 [95\% CI 1.15 to 1.31]). Again, these associations were significantly and numerically higher in women compared to men for both allcause mortality (1.33 [95\% CI 1.24 to 1.42$]$ versus 1.08 [1.01 to 1.15$])$ and cardiovascular death (1.44 [95\% CI 1.31 to 1.58] versus 1.10 [1.00 to 1.20], $p$ value for interaction $<0.001$ ) [39]. Eggers et al. observed numerically higher associations between high-sensitivity cardiac troponin I and death or incident cardiovascular disease in women compared to men in 1004 (502 women) elderly persons; however, sex was not a significant effect modifier for either [40].

These studies have important implications for clinical practice in guiding whether to adopt sex-specific thresholds for cardiac troponin. One of the key obstacles, to date, has been the lack of evidence that troponin concentrations above and below sex-specific cut points infer different prognostic information to that of single thresholds. Indeed, if higher troponin levels do infer worse prognosis for women, as some of the larger cohort studies seem to suggest, adopting single troponin thresholds is likely to misclassify some women as low risk with potentially important clinical ramifications.

\section{Sex Differences in the Diagnosis of Myocardial Infarction}

Whilst the Universal Definition of Myocardial Infarction acknowledges that sex-specific differences exist in the upper reference limits for these assays, no firm recommendations for practice were made in the latest iteration [41]. Since the publication of this guideline, several studies have evaluated the impact of sex-specific thresholds using high-sensitivity assays on the diagnosis of myocardial infarction (Table 2) $[42 \cdot \bullet, 43,44,46,47,48 \bullet \bullet]$.

In a prospective cohort of selected patients with suspected acute coronary syndrome, Mueller-Hennessen et al. reported that age-specific rather than sex-specific thresholds influenced the diagnosis of myocardial infarction using the high-sensitivity cardiac troponin T assay. Across 1282 patients (477 [37\%] women) with suspected acute coronary syndrome evaluated using a highsensitivity troponin $\mathrm{T}$ assay, the use of sex-specific compared to uniform thresholds changed the rates of myocardial infarction from 16 to $22 \%$ in women and from 23 to $21 \%$ in males. However, the use of sex-specific thresholds had minimal impact on risk stratification for death at 1 or 3 months [45]. However, Rubini-Gimenez et al., in 2734 patients (876 [32\%] women), did not observe any effect of sex-specific thresholds on the diagnosis of myocardial infarction and concluded that a uniform threshold should remain standard of care for the high-sensitivity cardiac troponin $\mathrm{T}$ assay [46].

In contrast, two studies have shown that women with suspected acute coronary syndrome identified by the highsensitivity cardiac troponin I assay with sex-specific thresholds, but missed with uniform thresholds, are at higher risk of

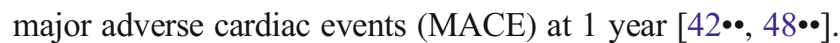
Cullen et al. demonstrate in a multi-centre cohort study of 2841 patients that MACE was increased in women with troponin concentrations above the sex-specific diagnostic 


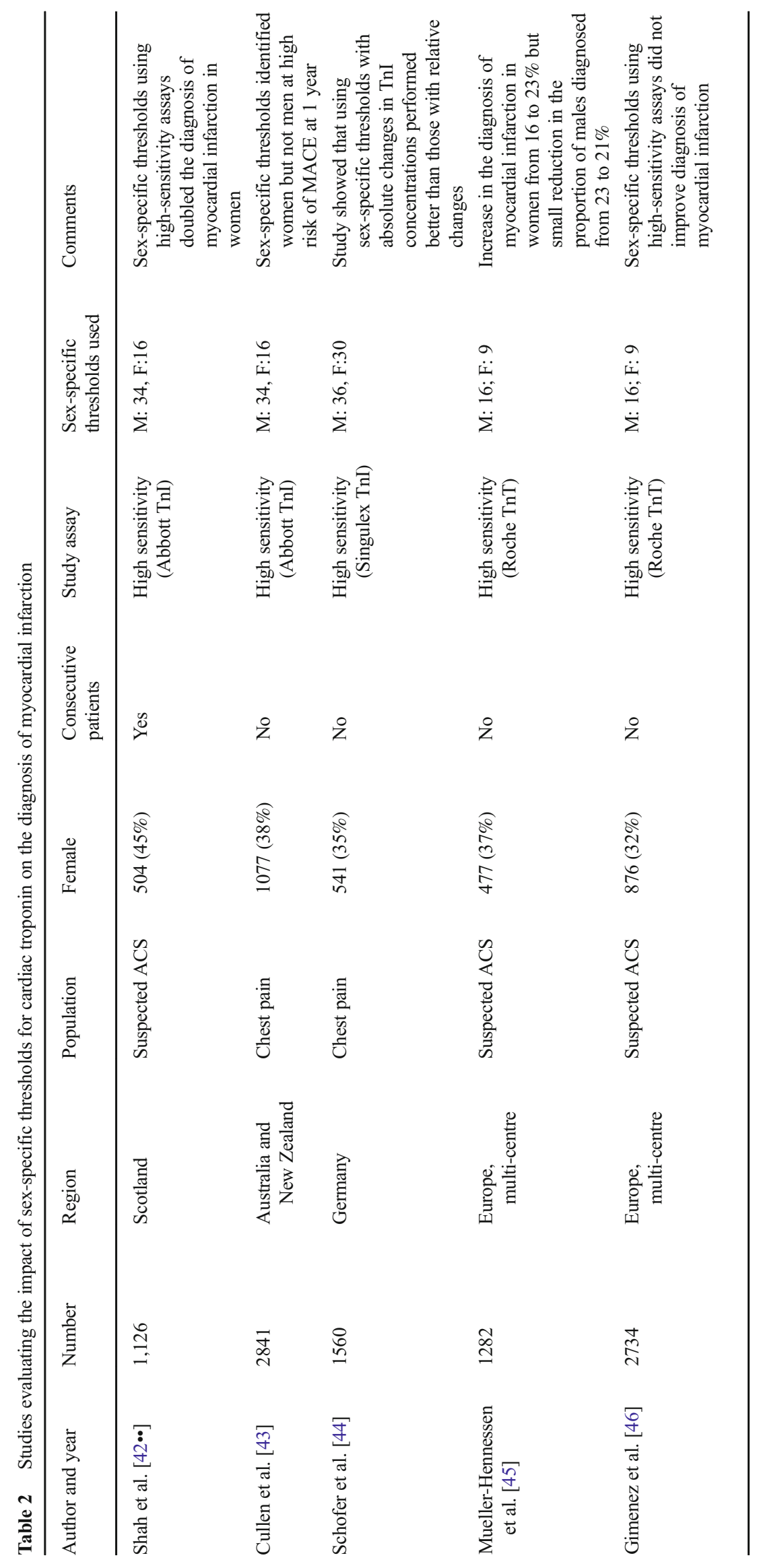


threshold, but below the uniform threshold, and that this association persisted following adjustment for age. Similar findings were reported in a consecutive cohort of 1126 patients with suspected acute coronary syndrome from a single site in Scotland [ $42 \bullet \bullet]$. This study showed that the use of sex-specific thresholds almost doubled the diagnosis of myocardial infarction in women when compared to a contemporary assay using a uniform threshold.

Finally, it is important to note that there were marked differences in the proportion of women recruited into these cohort studies, and therefore, it is likely that selection bias by gender explains some of the discrepancies observed. The proportion of women included in selected patient cohorts varied from 32 to $38 \%$, but in consecutive patient cohorts, where selection bias is minimised, the proportion of women was higher at $45 \%$ (Table 2).

\section{Methodological Limitations of Studies Evaluating Sex-Specific Thresholds}

Unfortunately, the interpretation of diagnostic accuracy studies using high-sensitivity cardiac troponins is challenging. Cardiac troponin is integral to the diagnosis of myocardial infarction, and there is no independent gold standard test to define spontaneous or type 1 myocardial infarction, i.e. myocardial infarction due to plaque rupture and coronary thrombosis [49]. As such, claims that a more sensitive troponin test, whether using uniform or sexspecific thresholds, is better than the reference test may be contested $[49,50 \bullet]$. The results of these studies are highly influenced by choice of the reference test used to adjudicate the diagnosis. Furthermore, there are important differences between the patients recruited into these cohort studies and those in whom testing is performed in clinical practice. Troponin testing is performed widely in patients attending the Emergency Department, where clinicians are required to rapidly evaluate and exclude multiple potential diagnosis simultaneously. The performance of high-sensitivity cardiac troponin testing is likely to differ here compared to selected patients presenting with symptoms of suspected acute coronary syndrome alone. Women are less likely to be recruited as they are thought to present with less typical symptoms and are older and more likely to have comorbid conditions. As such, selection bias will influence the generalisability of the findings from diagnostic accuracy studies. Due to these limitations, any review of studies comparing diagnostic performance of uniform and sex-specific thresholds requires careful attention to methodology.

When evaluating the use of more sensitive tests and cut points that are lower than those measurable by previous tests, the new test detects additional cases of apparent disease. There will always be uncertainty as to whether those patients identified by the new test should be classified or treated in the same way: Are these additional patients really true positives? This is particularly relevant when retrospectively evaluating the use of sex-specific thresholds as the 99th centile upper reference limit in women is below the uniform threshold used to guide further investigation at the time of presentation. In contrast, in men, the sex-specific 99th centile upper reference limit is higher than the uniform threshold. As such, women identified retrospectively as having elevated cardiac troponin concentrations only when sex-specific thresholds are applied often did not undergo further diagnostic investigations at the time of presentation to inform diagnostic adjudication. Furthermore, women are under-represented in these cohort studies as, unlike their male counterparts, they were less likely to be identified as having an acute coronary syndrome at the time of their presentation.

Glasziou et al. propose an elegant way of addressing this issue: the use of an 'umpire test' [50•0]. They suggest a threestep process. First, investigators need to highlight cases, which are discordant and identified only by one of the tests. Second, they propose the use of an independent and fair test that is unconditional to either of the tests being evaluated: the umpire test. Third, these 'umpire tests' can include a multitude of variables including causal exposures, outcomes and treatment responses [50••]. For example, evidence of myocardial ischaemia on electrocardiography could be used as such an umpire test, where the discrepant group with a higher prevalence of ischaemia would indicate the superior test.

We adapted this methodology in our cohort of patients with suspected acute coronary syndrome [42••]. The analysis showed that those women newly identified as having myocardial infarction using sex-specific thresholds had baseline characteristics, risk factor profiles and prognoses similar to those patients identified as having myocardial infarction using a uniform threshold, and that they were distinct from those identified as not having myocardial infarction using both approaches. Based on a logistic regression model, we showed that those patients reclassified using sex-specific thresholds followed a similar probability distribution and clinical trajectory to those patients classified as myocardial infarction using the uniform threshold [42••]. Importantly, this analysis was conducted in a cohort that included all consecutive patients without selection bias, and therefore, a similar proportion of men and women were evaluated. We conclude that sex-specific thresholds for high-sensitivity cardiac troponin I identify a significant proportion of women missed using uniform thresholds and that these women have other clinical features of myocardial infarction and are at high risk of recurrent events.

\section{Implementation and Clinical Practice}

Lowering the diagnostic threshold following implementation of a more sensitive troponin assay has been shown to improve clinical outcomes $[51 \bullet \cdot]$. There was a halving of recurrent myocardial infarction and death in those patients with small previously unrecognised increases in cardiac troponin 
concentration. These improvements in outcome were associated with better patient care including more specialist referrals, investigations and better provision of evidenced-based therapies [51••]. These observations highlight the importance that clinicians place on an abnormal troponin measurement in guiding clinical decisions and suggest that high-sensitivity troponin assays with even lower diagnostic thresholds have the potential to improve care further.

However, progressively lowering the threshold of plasma troponin concentration in order to define increasing numbers of patients with myocardial infarction is likely to increase sensitivity at the expense of reduced specificity [52]. Elevated cardiac troponin is common in patients without acute coronary syndrome and accounts for up to $30 \%$ of all patients with troponin concentrations above the upper reference limit [53, 54]. Furthermore, lowering the diagnostic threshold for cardiac troponin disproportionately increases the detection of patients with elevated cardiac troponin levels in patients without acute coronary syndrome. Outcomes of these patients remain poor after implementation and does not appear to be modifiable [55]. High-sensitivity cardiac troponin assays are likely to increase the frequency of troponin elevations in non-acute coronary syndrome pathologies especially in women if sex-specific thresholds are employed. In women, the proportion with troponin elevations due to non-acute coronary syndrome pathologies increased from 7 to $12 \%$, moving from a less sensitive contemporary troponin assay to a highsensitivity assay with sex-specific thresholds [42••]. Whether implementation of high-sensitivity cardiac troponin assays and use of sex-specific diagnostic thresholds improve outcomes through better targeting of treatments for coronary heart disease are the focus of an ongoing multi-centre randomised control trial (clinical trials.gov NCT01852123).

\section{Conclusion}

The evidence for and against the use of sex-specific thresholds when implementing high-sensitivity cardiac troponin assays is evolving. There are three main arguments in favour of adopting sex-specific thresholds. First, cardiac troponin concentrations are consistently lower in women than men, across multiple populations from different ethnic backgrounds. Second, cardiac troponin concentrations in stable populations infer different prognostic information in men and women with stronger associations noticed in women when uniform thresholds are applied. It is clear that lower thresholds are needed for women to provide comparable risk stratification. Third, in the setting of suspected acute coronary syndrome, sex-specific thresholds for cardiac troponin I identify women at high risk of future myocardial infarction and death. Finally, it is recognised that women are less likely to receive evidence-based therapy and have worse outcomes in acute coronary syndromes. The use of sex-specific diagnostic criteria may help to address these inequalities. Given these discrepancies in the quality of care provided for men and women, it seems axiomatic that sex-specific criteria be employed in the diagnosis of myocardial infarction.

Acknowledgements Nicholas L. Mills is supported by the Butler Senior Clinical Research Fellowship (FS/16/14/32023) from the British Heart Foundation.

Funding This study is supported by the British Heart Foundation Special Project Grant (SP/12/10/29922), Butler Senior Clinical Research Fellowship (FS/16/14/32023).

\section{Compliance with Ethical Standards}

Conflict of Interest Anoop S.V. Shah has received honoraria from Abbott Diagnostics. Amy V. Ferry declares that she has no conflict of interest. Nicholas L. Mills has consulted and received research grants from Abbott Diagnostics and has consulted for Roche Diagnostics and Singulex.

Human and Animal Rights and Informed Consent All studies included in this review were performed in accordance with the ethical standards of the institutional and/or national research committee and with the 1964 Helsinki declaration and its later amendments or comparable ethical standards.

Open Access This article is distributed under the terms of the Creative Commons Attribution 4.0 International License (http:// creativecommons.org/licenses/by/4.0/), which permits unrestricted use, distribution, and reproduction in any medium, provided you give appropriate credit to the original author(s) and the source, provide a link to the Creative Commons license, and indicate if changes were made.

\section{References}

Papers of particular interest, published recently, have been highlighted as:

•- Of major importance

1. Lozano R, Naghavi M, Foreman K, Lim S, Shibuya K, Aboyans V, et al. Global and regional mortality from 235 causes of death for 20 age groups in 1990 and 2010: a systematic analysis for the Global Burden of Disease Study 2010. Lancet. 2012;380(9859):2095-128.

2.• Wenger NK. Women and coronary heart disease: a century after herrick : understudied, underdiagnosed, and undertreated. Circulation. 2012;126:604-11. Manuscript highlighting sex differences in the evaluation of patients with acute coronary syndrome.

3. Canto JG, Rogers WJ, Goldberg RJ, Peterson ED, Wenger NK, Vaccarino $\mathrm{V}$, et al. Association of age and sex with myocardial infarction symptom presentation and in-hospital mortality. JAMA. 2012;307(8):813-22.

4. Mehta LS, Beckie TM, DeVon HA, Grines CL, Krumholz HM, Johnson MN, et al. Acute myocardial infarction in women: a scientific statement from the American Heart Association. Circulation. 2016;133(9):916-47.

5. Vaccarino V, Parsons L, Every NR, Barron HV, Krumholz HM. Sex-based differences in early mortality after myocardial infarction. National Registry of Myocardial Infarction 2 Participants. New Eng J Med. 1999;341(4):217-25. 
6. Becker RC, Terrin M, Ross R, Knatterud GL, Desvigne-Nickens P, Gore JM, et al. Comparison of clinical outcomes for women and men after acute myocardial infarction. The Thrombolysis in Myocardial Infarction Investigators. Ann Intern Med. 1994;120(8):638-45.

7. Woodfield SL, Lundergan CF, Reiner JS, Thompson MA, Rohrbeck SC, Deychak Y, et al. Gender and acute myocardial infarction: is there a different response to thrombolysis? J Am Coll Cardiol. 1997;29(1):35-42.

8. Lincoff AM, Califf RM, Ellis SG, Sigmon KN, Lee KL, Leimberger JD, et al. Thrombolytic therapy for women with myocardial infarction: is there a gender gap? Thrombolysis and Angioplasty in Myocardial Infarction Study Group. J Am Coll Cardiol. 1993;22(7):1780-7.

9. Malacrida R, Genoni M, Maggioni AP, Spataro V, Parish S, Palmer A, et al. A comparison of the early outcome of acute myocardial infarction in women and men. The Third International Study of Infarct Survival Collaborative Group. N Engl J Med. 1998;338(1):8-14.

10. Alpert JS, Thygesen K, Jaffe A, White HD. The universal definition of myocardial infarction: a consensus document: ischaemic heart disease. Heart. 2008;94(10):1335-41.

11. Adams 3rd JE, Abendschein DR, Jaffe AS. Biochemical markers of myocardial injury. Is MB creatine kinase the choice for the 1990s? Circulation. 1993;88(2):750-63.

12. Apple FS, Quist HE, Doyle PJ, Otto AP, Murakami MM. Plasma 99th percentile reference limits for cardiac troponin and creatine kinase MB mass for use with European Society of Cardiology/ American College of Cardiology consensus recommendations. Clin Chem. 2003;49(8):1331-6.

13. Morrow DA, Cannon CP, Jesse RL, Newby LK, Ravkilde J, Storrow $\mathrm{AB}$, et al. National Academy of Clinical Biochemistry Laboratory Medicine Practice Guidelines: clinical characteristics and utilization of biochemical markers in acute coronary syndromes. Circulation. 2007;115(13):e356-75.

14. Coudrey L. The troponins. Arch Intern Med. 1998;158(11):1173-80.

15. Katus HA, Looser S, Hallermayer K, Remppis A, Scheffold T, Borgya A, et al. Development and in vitro characterization of a new immunoassay of cardiac troponin T. Clin Chem. 1992;38(3): 386-93.

16. Thygesen K, Alpert JS, Jaffe AS, Simoons ML, Chaitman BR, White HD, et al. Third universal definition of myocardial infarction. Circulation. 2012;126(16):2020-35.

17. Apple FS, Collinson PO. Analytical characteristics of highsensitivity cardiac troponin assays. Clin Chem. 2012;58(1):54-61.

18. Hamm CW, Ravkilde J, Gerhardt W, Jorgensen P, Peheim E, Ljungdahl $\mathrm{L}$, et al. The prognostic value of serum troponin $\mathrm{T}$ in unstable angina. N Engl J Med. 1992;327(3):146-50.

19. Wiviott SD, Cannon CP, Morrow DA, Murphy SA, Gibson CM, $\mathrm{McCabe} \mathrm{CH}$, et al. Differential expression of cardiac biomarkers by gender in patients with unstable angina/non-ST-elevation myocardial infarction: a TACTICS-TIMI 18 (Treat Angina with Aggrastat and determine Cost of Therapy with an Invasive or Conservative Strategy-Thrombolysis In Myocardial Infarction 18) substudy. Circulation. 2004;109(5):580-6.

20. Apple FS, Ler R, Murakami MM. Determination of 19 cardiac Troponin I and T Assay 99th percentile values from a common presumably healthy population. Clin Chem. 2012;58(11):1574-81.

21. Giannitsis E, Kurz K, Hallermayer K, Jarausch J, Jaffe AS, Katus HA. Analytical validation of a high-sensitivity cardiac troponin T assay. Clin Chem. 2010;56(2):254-61.

22. Saenger AK, Beyrau R, Braun S, Cooray R, Dolci A, Freidank H, et al. Multicenter analytical evaluation of a high-sensitivity troponin T assay. Clin Chim Acta. 2011;412(9-10):748-54.

23. Koerbin G, Tate JR, Hickman PE. Analytical characteristics of the Roche highly sensitive troponin $\mathrm{T}$ assay and its application to a cardio-healthy population. Ann Clin Biochem. 2010;47(Pt 6):524-8.
24. Collinson PO, Heung YM, Gaze D, Boa F, Senior R, Christenson $\mathrm{R}$, et al. Influence of population selection on the 99th percentile reference value for cardiac troponin assays. Clin Chem. 2012;58(1):219-25.

25. Mingels A, Jacobs L, Michielsen E, Swaanenburg J, Wodzig W, van Dieijen-Visser M. Reference population and marathon runner sera assessed by highly sensitive cardiac troponin T and commercial cardiac troponin T and I assays. Clin Chem. 2009;55(1):101-8.

26. Koerbin G, Tate J, Potter JM, Cavanaugh J, Glasgow N, Hickman PE. Characterisation of a highly sensitive troponin I assay and its application to a cardio-healthy population. Clin Chem Lab Med. 2012;50(5):871-8.

27.• Apple FS, Simpson PA, Murakami MM. Defining the serum 99th percentile in a normal reference population measured by a highsensitivity cardiac troponin I assay. Clin Biochem. 2010;43(12):

1034-6. Comprehensive analysis to evaluate the 99th centile diagnostic threshold across five high-sensitivity troponin assays using the same cohort.

28. McKie PM, Heublein DM, Scott CG, Gantzer ML, Mehta RA, Rodeheffer RJ, et al. Defining high-sensitivity cardiac troponin concentrations in the community. Clin Chem. 2013;59(7):1099-107.

29. de Lemos JA, Drazner MH, Omland T, Ayers CR, Khera A, Rohatgi A, et al. Association of troponin T detected with a highly sensitive assay and cardiac structure and mortality risk in the general population. JAMA. 2010;304(22):2503-12.

30. White HD. Pathobiology of troponin elevations: do elevations occur with myocardial ischemia as well as necrosis? J Am Coll Cardiol. 2011;57(24):2406-8.

31. Motiwala SR, Sarma A, Januzzi JL, O’Donoghue ML. Biomarkers in ACS and heart failure: should men and women be interpreted differently? Clin Chem. 2014;60(1):35-43.

32. de Simone G, Devereux RB, Daniels SR, Meyer RA. Gender differences in left ventricular growth. Hypertension. 1995;26(6 Pt 1):979-83.

33. Donaldson C, Eder S, Baker C, Aronovitz MJ, Weiss AD, HallPorter M, et al. Estrogen attenuates left ventricular and cardiomyocyte hypertrophy by an estrogen receptor-dependent pathway that increases calcineurin degradation. Circ Res. 2009;104(2):265-75.

34. Robb AO, Mills NL, Din JN, Cameron S, Ludlam CA, Newby DE, et al. Acute endothelial tissue plasminogen activator release in pregnancy. J Thromb Haemost. 2009;7(1):138-42.

35. Lansky AJ, Ng VG, Maehara A, Weisz G, Lerman A, Mintz GS, et al. Gender and the extent of coronary atherosclerosis, plaque composition, and clinical outcomes in acute coronary syndromes. JACC Cardiovasc Imag. 2012;5(3 Suppl):S62-72.

36. Vaccarino V, Badimon L, Corti R, de Wit C, Dorobantu M, Manfrini O, et al. Presentation, management, and outcomes of ischaemic heart disease in women. Nat Rev Cardiol. 2013;10(9):508-18.

37. Giannitsis E. Sex-specific troponin measures for diagnosis of acute coronary syndrome. Heart. 2016;102(2):91-2.

38. Dallmeier D, Denkinger M, Peter R, Rapp K, Jaffe AS, Koenig W, et al. Sex-specific associations of established and emerging cardiac biomarkers with all-cause mortality in older adults: the ActiFE study. Clin Chem. 2015;61(2):389-99.

39. Omland T, de Lemos JA, Holmen OL, Dalen H, Benth JS, Nygard $\mathrm{S}$, et al. Impact of sex on the prognostic value of high-sensitivity cardiac troponin I in the general population: the HUNT study. Clin Chem. 2015;61(4):646-56.

40. Eggers KM, Johnston N, Lind L, Venge P, Lindahl B. Cardiac troponin I levels in an elderly population from the communitythe implications of sex. Clin Biochem. 2015;48(12):751-6.

41. Thygesen K, Alpert JS, Jaffe AS, Simoons ML, Chaitman BR, White HD, et al. Third universal definition of myocardial infarction. Eur Heart J. 2012;33(20):2551-67.

42.• Shah AS, Griffiths M, Lee KK, McAllister DA, Hunter AL, Ferry $\mathrm{AV}$, et al. High sensitivity cardiac troponin and the under-diagnosis of myocardial infarction in women: prospective cohort study. BMJ. 
2015;350:g7873. First analysis to evaluate sex-specific thresholds with high-sensitivity cardiac troponin assays for the diagnosis of myocardial infarction.

43. Wildi K, Gimenez MR, Twerenbold R, Reichlin T, Jaeger C, Heinzelmann A, et al. Misdiagnosis of myocardial infarction related to limitations of the current regulatory approach to define clinical decision values for cardiac troponin. Circulation. 2015;131(23): 2032-40.

44. Schofer N, Brunner FJ, Schluter M, Ojeda F, Zeller T, Baldus S, et al. Gender-specific diagnostic performance of a new highsensitivity cardiac troponin I assay for detection of acute myocardial infarction. Eur Heart J Acute Cardiovasc Care. 2016.

45. Mueller-Hennessen M, Lindahl B, Giannitsis E, Biener M, Vafaie M, de Filippi CR. Diagnostic and prognostic implications using age- and gender-specific cut-offs for high-sensitivity cardiac troponin T-sub-analysis from the TRAPID-AMI study. Int J Cardiol. 2016;209:26-33.

46. Gimenez RM, Twerenbold R, Boeddinghaus J, Nestelberger T, Puelacher C, Hillinger P, et al. Clinical effect of sex-specific cutoff values of high-sensitivity cardiac troponin $\mathrm{T}$ in suspected myocardial infarction. JAMA Cardiol. 2016;1(8):912-20.

47. Shah AS, Newby DE, Mills NL. High sensitivity cardiac troponin in patients with chest pain. BMJ. 2013;347:f4222.

48.• Cullen L, Greenslade JH, Carlton EW, Than M, Pickering JW, Ho A, et al. Sex-specific versus overall cut points for a high sensitivity troponin I assay in predicting 1-year outcomes in emergency patients presenting with chest pain. Heart. 2016;102(2):120-6. Analysis demonstrating that sex-specific thresholds for highsensitivity cardiac troponin improved the identification of patients with poor long-term outcomes.

49. Jaffe AS, Apple FS, Morrow DA, Lindahl B, Katus HA. Being rational about (im)precision: a statement from the Biochemistry
Subcommittee of the Joint European Society of Cardiology/ American College of Cardiology Foundation/American Heart Association/World Heart Federation Task Force for the definition of myocardial infarction. Clin Chem. 2010;56(6):941-3.

50.• Glasziou P, Irwig L, Deeks JJ. When should a new test become the current reference standard? Ann Intern Med. 2008;149(11):816-22. Review article explaining the limitations of diagnostic accuracy studies in disease states where a diagnostic gold standard is absent.

51.• Mills NL, Churchhouse AM, Lee KK, Anand A, Gamble D, Shah AS, et al. Implementation of a sensitive troponin I assay and risk of recurrent myocardial infarction and death in patients with suspected acute coronary syndrome. JAMA. 2011;305(12):1210-6. First analysis to demonstrate that lowering the diagnostic threshold for myocardial infarction was associated with improved clinical outcomes in patients with suspected acute coronary syndrome.

52. Jaffe AS. TRAPID or trapped? Ann Emerg Med. 2016;68(1): 88-91.

53. Saaby L, Poulsen TS, Diederichsen AC, Hosbond S, Larsen TB, Schmidt $\mathrm{H}$, et al. Mortality rate in type 2 myocardial infarction: observations from an unselected hospital cohort. Am J Med. 2014;127(4):295-302.

54. Saaby L, Poulsen TS, Hosbond S, Larsen TB, Pyndt Diederichsen $\mathrm{AC}$, Hallas J, et al. Classification of myocardial infarction: frequency and features of type 2 myocardial infarction. Am J Med. 2013;126(9):789-97.

55. Shah AS, McAllister DA, Mills R, Lee KK, Churchhouse AM, Fleming KM, et al. Sensitive troponin assay and the classification of myocardial infarction. Am J Med. 2015;128(5):493-501. e3.

56. Aw TC, Phua SK, Tan SP. Measurement of cardiac troponin I in serum with a new high-sensitivity assay in a large multi-ethnic Asian cohort and the impact of gender. Clin Chim Acta. 2013. 